\title{
Traumatismo craneoencefálico en pacientes con síndrome psicótico: etiopatogenia y estatus nosológico.
}

Traumatic brain injury in patients with psychotic syndrome: etiopathogenesis and nosological status.

\author{
Álvaro Frías Ibáñez a , Carolina Palma Sevillano ${ }^{\text {b }}$, Nuria Farriols Hernando ${ }^{\mathrm{c}}$. \\ ${ }^{a, b y c}$ Psicólogo Clínico. Centro de Salud Mental de Adultos. Servicio de psiquiatría. Hospital de Mataró, \\ Barcelona, España.
}

Correspondencia: Álvaro Frías Ibáñez,(afrias@csdm.cat)

Recibido: 02/10/2014; aceptado: 04/05/2015

\begin{abstract}
RESUMEN: Introducción. Uno de los debates nosológicos más controvertidos en el ámbito de la Neuropsiquiatría versa sobre el diagnóstico diferencial entre psicosis adquiridas e idiopáticas"(esquizofrenia) en pacientes que previamente han sufrido un traumatismo craneoencefálico (TCE). Caso clínico. Se expone el caso de un varón de 15 años, sin antecedentes psiquiátricos personales ni familiares, que a los 5 meses de sufrir un TCE con pérdida de conciencia desarrolló un síndrome psicótico crónico caracterizado por sintomatología positiva, siendo ésta resistente a todos los tratamientos psicofarmacológicos prescritos. No se evidenció lesión cerebral focalizada a través de neuroimagen estructural. Discusión. El TCE puede actuar como agente etiopatogénico primario (psicosis debida a un TCE) o precipitante (esquizofrenia) en los cuadros psicóticos manifestados. Los dos criterios externos que contribuyen en mayor medida a la distinción de ambos trastornos son la escasa sintomatología negativa y la baja vulnerabilidad genética entre las personas con psicosis debida a un TCE. Con relativa frecuencia, tanto el periodo de latencia TCE-psicosis como los correlatos neurocognitivos y neurobiológicos asociados apenas permiten esclarecer el estatus nosológico del síndrome psicótico. Son necesarios ensayos clínicos controlados para valorar la eficacia de los antipsicóticos en aquellos sujetos con psicosis debida a un TCE.

PALABRAS CLAVE: Esquizofrenia, diagnóstico diferencial, nosología, psicosis, tratamiento, traumatismo craneoencefálico.
\end{abstract}

ABSTRACT: Introduction. One of the most controversial nosological issues in neuropsychiatry deals with the differential diagnosis between acquired and idiopathic psychosis (schizophrenia) in patients who have previously undergone a traumatic brain injury (TBI). Case report. A 15 year-old man, without personal or family psychiatric history, developed a chronic syndrome characterized by positive psychotic symptoms 5 months after suffering a TBI with loss of consciousness. The syndrome was refractory to all psychopharmacological treatments prescribed. There was no brain alteration detected through structural imaging. Discussion. TBI may play a role either as primary etiopathogenic agent (psychosis due to TBI) or as triggering factor (schizophrenia) in the development of psychotic symptoms. Fewer negative symptoms as well as lower genetic vulnerability contribute to differentiate the former group from the latter one. Usually, neither the latency period between TBI-psychosis onset nor the neurocognitive and neurobiological correlates allow to clarify the nosological status. Further controlled clinical trials are needed to assess the efficacy of antipsychotics in those subjects with psychosis due to TBI.

KEY WORDS: Schizophrenia, differential diagnosis, nosology, psychosis, treatment, traumatic brain injury. 


\section{Introducción}

La presencia de un traumatismo craneoencefálico (TCE) entre los antecedentes personales de un sujeto que desarrolla un síndrome psicótico plantea al profesional el interrogante acerca del papel que este factor pudiera haber ejercido en la génesis de dicho trastorno mental, determinando en última instancia el estatus nosológico del cuadro clínico.

\section{Motivo de consulta}

Se expone el caso de un varón de 35 años que ingresa en una unidad psiquiátrica de larga estancia ante la imposibilidad de manejo ambulatorio en el centro de Salud Mental de referencia. Es derivado por un cuadro psicótico de 20 años de evolución, refractario a todos los tratamientos pautados hasta ese momento.

\section{Historia del problema}

La clínica psicótica se inició a los 15 años, 5 meses después de sufrir un TCE cerrado aparentemente accidental, siendo éste localizado en el área temporal derecha y yendo acompañado de pérdida de conciencia de duración no precisada (varios minutos). Entre sus características psicopatológicas destacaba la presencia de un discurso totalmente disgregado, con predominio de delirios no sistematizados (paranoides, de control, filiativos y nihilistas), así como alucinaciones visuales y auditivas que, en conjunto, generaban heteroagresividad física hacia el resto de miembros de la familia. Así mismo, el paciente manifestaba afectividad inadecuada y comportamiento desorganizado, cursando con nula conciencia de enfermedad y escaso deterioro de las funciones cognoscitivas elementales (atención, memoria, etc.). Recibió un diagnóstico primario de "esquizofrenia indiferenciada". Durante los 19 años de seguimiento ambulatorio no se obtuvo en ningún período una remisión parcial de su psicopatología basal, mostrando una pobre adherencia y respuesta a los tratamientos psicofarmacológicos prescritos, lo que a larga motivó varias hospitalizaciones, así como una elevada sobrecarga familiar. Abusó ocasionalmente del alcohol y cannabis en dicho período. Las pruebas médicas llevadas a cabo (EEG, RMN) no hallaron ninguna etiología orgánica focalizada. 


\section{Psicobiografia}

Fue fruto de un parto y puerperio sin incidencias. Desarrollo psicomotor, control de esfínteres y aprendizaje lecto-escritor dentro de la normalidad. No hay descritas reacciones adversas medicametosas ni alergias conocidas. Descrito desde la infancia como un niño con un carácter extravertido. En la escuela tuvo un rendimiento académico bajo, por lo que desde los 14 años ayudó en el negocio familiar. Buena red de apoyo sociofamiliar. No constan antecedentes (neuro)psiquiátricos personales ni familiares.

\section{Exploración clínica}

Durante la entrevista de ingreso en la unidad de larga estancia se hallaba consciente y vigil. Aspecto e higiene descuidada. Orientado en tiempo, lugar y persona. Pensamiento desorganizado, con lenguaje disgregado y pararrespuestas. El contenido destacaba por ideas delirantes extravagantes y de perjuicio no sistematizadas, acompañadas de una destacable inadecuación afectiva. Presentaba continuas alucinaciones visuales y auditivas, con abundantes soliloquios y conductas en apariencia apragmáticas. Sin alteraciones en la esfera instintiva. Nula conciencia de enfermedad.

\section{Diagnóstico}

Psicosis orgánica postraumática F06.8

\section{Tratamiento y evolución}

Durante los 6 años que lleva ingresado, el cuadro psicótico ha persistido sin ningún período de remisión parcial, a pesar de las modificaciones introducidas en los tratamientos prescritos. No se ha evidenciado sintomatología negativa concomitante. En la actualidad es tratado con Clozapina 100 2-2-2 y Aripiprazol 15 1-1-1.

El paciente es autónomo para la ejecución de las actividades básicas de la vida diaria (ABVD), sin haberse constatado un deterioro cognitivo manifiesto (mini-mental: 29/35). Muestra un comportamiento adaptado a las normas de funcionamiento de la unidad, manteniendo una relación cordial con el resto de pacientes y personal sanitario. 
En ocasiones puntuales ha sido necesario controlar su comportamiento desorganizado (parafilias). Respecto a las tareas de rehabilitación cognitiva, apenas ha obtenido beneficio de aquellos talleres que requieren procesamiento cognitivo (p.e. terapia psicológica integrada) debido a su pensamiento desorganizado. No obstante, ha participado en tareas extrahospitalarias supervisadas por monitores ocupacionales.

\section{Discusión}

Una de las mayores controversias en el campo de la Neuropsiquiatría versa sobre el diagnóstico diferencial entre psicosis adquiridas e idiopáticas en pacientes con TCE previo. En términos generales, se sostiene que la prevalencia de síndromes psicóticos en personas que han sufrido un TCE es mucho mayor que la observada en población general (1). Sin embargo, la asociación entre trauma cerebral y psicosis no es unívoca, habiéndose postulado varias teorías explicativas sobre ello. Por una parte, el TCE podría ser un factor precipitante de una psicosis funcional (esquizofrenia) en sujetos con predisposición genética (2), aumentando el trauma el riesgo relativo en relación a otros familiares vulnerables (3-4), principalmente a través de cambios en la neuroplasticidad cerebral (5). Por otro lado, el TCE podría ser la causa directa o primaria de una psicosis "orgánica exógena" (schizophrenia-like psychosis) en ausencia de antecedentes familiares, caracterizándose generalmente este síndrome psicótico por una ínfima presencia de síntomas negativos (6-7), así como por déficits neuropsicológicos (funciones ejecutivas y memoria verbal), estructurales (frontotemporales) o neuroquímicos (acetilcolina) similares, aunque menos generalizados, a los hallados en pacientes esquizofrénicos $(1,8-9)$. Por último, la asociación entre TCE y psicosis (esquizofrenia) podría ser espúrea o inversa, siendo realmente el segundo un factor de riesgo para el primero, especialmente durante las fases prodrómicas en las que predomine agitación psicomotriz (10).

Sobre la base de estas hipótesis planteadas, el caso que nos compete puede quedar integrado dentro de aquellos trastornos psicóticos originados de manera primaria por una condición médica (TCE). Desde un punto de vista nosotáxico, este tipo de patología es recogida con el diagnóstico de "trastorno psicótico debido a TCE" (DSM-V) o "psicosis orgánica postraumática" (CIE-10). La prevalencia de este trastorno es escasa, variando su ratio (4-8\%) en función del tipo de muestra y los criterios utilizados en la operativización del constructo "psicosis". Distintos factores de riesgo han sido reconocidos como variables predictoras de estas psicosis postraumáticas (versus TCE sin psicosis), a saber: antecedentes neurológicos congénitos / adquiridos (p.e. otro TCE previo), una menor inteligencia general y la severidad del trauma objetivada a través de la duración de la pérdida de conciencia $(7,9)$. Respecto 
a la demora entre el TCE y el inicio de la psicosis, ésta puede ser variable, oscilando en una amplia horquilla entre los 6 meses y 5 años $(7,10)$, si bien más del 50 por cien de los casos se presentan en los dos primeros años. A nivel nosológico, dado que el tiempo de latencia TCE-psicosis adquirida puede ser dilatado, no se ha establecido de manera operativa ningún intervalo crítico al respecto.

En resumen, la presencia previa de un TCE no es indicador per se de la etiología y estatus nosológico de un síndrome psicótico. El clínico deberá valorar otros criterios clínicos (sintomatología negativa, antecedentes familiares) a la hora de considerar si el TCE pudiera estar actuando como un factor primario (psicosis postraumática) o precipitante (esquizofrenia) de dicho cuadro (11-12). Son necesarios ensayos clínicos controlados para valorar la eficacia de los antipsicóticos en esta clase de pacientes (13).

\section{BIBLIOGRÁFICAS}

(1) Bennouna M, Greene VB, Defranoux L. Cholinergic hypothesis in psychosis following traumatic brain injury and cholinergic hypothesis in schizophrenia: A link? Encephale 2007;33:61620 .

(2) Kim E. Does traumatic brain injury predispose individuals to develop schizophrenia? Curr Opin Psychiatry 2008;21:286-9.

(3) Abdel Malik P, Husted J, Chow EW, Bassett AS. Childhood head injury and expression of schizophrenia in multiply affected families. Arch Gen Psychiatry 2003;60:231-6.

(4) Malaspina D, Goetz RR, Friedman JH, Kaufmann CA, Faraone SV, Tsuang M, et al. Traumatic brain injury and schizophrenia in members of schizophrenia and bipolar disorder pedigrees. Am J Psychiatry 2001;158:440-6.

(5) Thomas G, Genest P, Walter M, Cozic JY. Can concepts of neurodevelopment and neuroplasticity explain post-traumatic psychoses? Neurochirurgie 2009;55:36-9.

(6) Fujii DE, Ahmed I. Characteristics of psychotic disorder due to traumatic brain injury: an analysis of case studies in the literature. J Neuropsychiatry Clin Neurosci 2002;14:130-40.

(7) Sachdev P, Smith JS, Cathcart S. Schizophrenia-like psychosis following traumatic brain injury: a chart-based descriptive and case-control study. Psychol Med 2001;31:231-9.

(8) Fuji DE, Ahmed I, Hishinuma E. A neuropsychological comparison of psychotic disorder following traumatic brain injury, traumatic brain injury without psychotic disorder, and schizophrenia. J Neuropsychiatry Clin Neurosci 2004;16:306-14.

(9) Fujii DE, Ahmed I. Risk factors in psychosis secondary to traumatic brain injury. J Neuropsychiatry Clin Neurosci 2001;13:61-9.

(10) Fujii DE, Ahmed I. Psychotic disorder following traumatic brain injury: A conceptual framework. Cogn Neuropsychiatry 2002;7:41-62.

(11) Fujii D, Fujii DC. Psychotic disorder due to traumatic brain injury: analysis of case studies in the literature. J Neuropsychiatry Clin Neurosci 2012;24:278-89. 
(12) Molloy C, Conroy RM, Cotter DR, Cannon M. Is traumatic brain injury a risk factor for schizophrenia? A meta-analysis of case-controlled population-based studies. Schizophr Bull 2011;37:1104-10.

(13) Viana B de M, Prais HA, Nicolato R, Caramelli P. Posttraumatic brain injury psychosis successfully treated with olanzapine. Prog Neuropsychopharmacol Biol Psychiatry 2010;34:233-5. 